\title{
THE RÔLE OF THE POINT-SET THEORY IN GEOMETRY AND DYNAMICS.
}

\section{PRESIDENTIAL ADDRESS DELIVERED BEFORE THE AMER- ICAN MATHEMATICAL SOCIETY, JANUARY $1,1915$.}

BY PRESIDENT E. B. VAN VLECK.

IN his "Child's Garden of Verses" Robert Louis Stevenson says simply but poetically:

"The world is so full of a number of things, I'm sure we should all be as happy as kings."

This catches the spirit of the Mengenlehre, and may well be taken as its motto. In more homely phrase the mathematician and physicist declare that the world is made up of an infinite number of things. Hence in thought and nature we have to consider numbers which are infinite. Even though they be not actually infinite, they are so enormously, unthinkably large that they can be most advantageously handled by regarding them as infinite. Such a mode of treatment was, of course, foreign to Greek thought. But, as you all know, the classic Greek mathematics was straitened by its failure to admit the infinitesimally small and the infinitely great magnitudes of the calculus. In somewhat similar fashion modern mathematics was long hampered by the lack of a mathematical theory, not of the potentially infinite quantity of the calculus, but of actually infinite aggregates. It was the incalculable and distinctive service of Georg Cantor to have perceived the urgent need of such a theory. Minerva-like and full panoplied it sprang from his teeming brain. Already its achievements have been very great, but it is far from maturity and its full powers are still to be revealed.

The influence of this new theory of infinite aggregates was first decisively felt in the theory of functions of the real and complex variables, where infinite sets of points, often irregular in character, frequently present themselves; for example, the discontinuities of an integrable function or the singularities of an analytic function, either of which may occur in infinite number. Here also, in the function-theory, its richest fruition 
has been attained. Three stages in the history of the Mengenlehre have been distinguished by Borel.* At first an offshoot or branch of the function theory, it soon reached a stage of independent existence where it was studied by and for itself. But its methods and ideas so rapidly permeated the functiontheory that a third period is distinguished by Borel, in which its essentials have been re-absorbed into the function-theory. The time would appear to be opportune for a general view of their mutual interaction, but inasmuch as this is so well-known in many of its aspects, $\dagger$ I have preferred to consider other bearings of Cantor's theory.

Quite in contrast with the definitive effects in the functiontheories is the plastic and vitalizing action of the Mengenlehre upon logic and philosophy. This may well be considered as beginning a fourth period, formally inaugurated at Paris last April in the first international congress for mathematical philosophy. Considered abstractly, Cantor's theory led inevitably to questions regarding the modes and soundness of reasoning upon infinite aggregates. Certain well-known antinomies resulted, demanding imperatively a solution. This has been generally sought by abandoning Cantor's definition of a set $\ddagger$ as any collection of definite and well-separated objects of perception or thought. For how otherwise can we make sure, as we certainly must, that there is no contradiction lurking in the set of objects brought together? By what, then, shall Cantor's definition be replaced? Must we confine ourselves to sets of objects each of which has been clearly defined in a finite number of words? The child begins to think on the world around him, undeterred by the consideration that the number of objects is limitless while he can reason in only a finite number of words. Numberless errors are made by him, the discovery and correction of which enhance his knowledge and power. Likewise in the early renaissance Cavalieri, Kepler, and others argued most imperfectly on indivisibles and infinites. But out of their thinking sprang ultimately the most powerful branch of mathematics. Undoubtedly great dangers lie hidden in reasoning on infinite classes of distinct objects whose elements have not all been

\footnotetext{
* Cf. Revue générale des Sciences, vol. 20 (1909), p. 315.

† Various aspects are well presented by Borel; loc. cit.

* "Unter einer Menge verstehen wir jede Zusammenfassung von bestimmten wohlunterschiedenen Objekten unserer Anschauung oder unseres Denkens zu einem Ganzen." Math. Ann., vol. 46 (1895), p. 481.
} 
defined and separated from one another by a finite number of words - for example, the points of a continuum. Principles may be used which subsequently will be discovered to be false, or at least more restrictedly true than had been imagined. But is this a sufficient cause for abandoning the so-called idealistic reasoning? Is it not rather a true mode of discovery? By boldly plunging forward in idealistic reasoning shall we not avoid the danger of falling into sterile rules of thought? Or, to the contrary, shall we thereby merely stumble into the pit we are trying to avoid? Is the Princip der Auswahl sound, or mere sound? Does mathematical reasoning involve principles which cannot be embraced in the syllogism, as claimed by Poincaré and denied by Russell; for instance, the principle of mathematical induction? Such questions as these, and many others, are merely an indication of the fermentation started by the Mengenlehre which has resulted in the re-creation of a department of mathematical logic and philosophy.

Into the consideration of this new and potent, but most abstract influence of the theory of aggregates I also do not propose to enter. My interest today lies neither in its best nor least formulated influence. It was the expectation of both Cantor and du Bois-Reymond that the theory of pointsets would become very useful in the field of mathematical physics. This expectation has been so little realized that even to the present time it is commonly said that no applications are there to be found. Though, indeed, as yet the applications to astronomy and physics are but few, they seem to me of great significance and promise. In fact, dynamics must always be deeply dependent for its development upon geometry, since dynamical trajectories are special cases of geometric curves. It seems to me, therefore, fitting to consider simultaneously: The service of the point-set theory to geometry and dynamics.

The rôle of any great branch of mathematics may be due to one or more of three causes,-its fundamental concepts, its distinctive methods, or its principal results. In the case of the point-set theory the rôle has been determined chiefly by its concepts and methods, which are intimately related. The greatness of a mathematical method or concept-for example, the notion of a group or of a differential coefficient-may be tested by its simplicity and universality. As has been so 
often said, the human intellect demands economy and convenience of thought. The scientist selects such principles as fulfil this demand and calls them true and fundamental. Not less imperative is it in mathematics to search for those concepts, methods, and theorems which are simple and widely applicable. This, let me urge parenthetically, is a lesson we may well ponder if our ambition for the greatness of American mathematics is to be attained. Judged by the same standard, the point-set theory is strong in both its methods and concepts, but preeminently in the latter.

To Georg Cantor was granted the rare fortune and merit to have uncovered more than one great concept. Some of his notions, as for instance the "power" of a set and the transfinite ordinal number of the first class, have as yet found their application almost wholly within the function-theory, and these I shall have no occasion to introduce. The concepts which I shall need are only five in number. Two of these are the familiar notions "limit point" and "everywhere dense," which antedate the work of Cantor. It was Cantor, however, who focused the search light upon the totality of the limit points of a given set, and called this totality the "derivative" or "first derived set" of the given set. The fourth concept is that of a "perfect set," which results when a set coincides with its derivative, so that it contains all its limit points and nothing but these. The fifth and last notion is perhaps Cantor's greatest concept, that of a "countable" or "enumerable" set, with which every one is familiar.* In particular, he showed the set of algebraic numbers to be countable.

On this concept of countability is based the modern analysis of the continuous interval, which lies at the bottom of ordinary geometry, be it euclidean or non-euclidean, two-dimensional or $n$-dimensional. Every well-informed mathematician knows how at last a satisfactory foundation for a continuous number system was discovered by Dedekind. His memoir of 1872 in which this was published is contemporary with Cantor's earliest publications relating to the Mengenlehre and may worthily be regarded with Cantor's work as the chief spring of our point-set theory. Starting with the system of rational numbers arranged in natural order of magni-

* A set is said to be countable when by proper selection we can count its elements, "one, two, three," etc., ad infinitum, omitting no element and reaching each element at some time in our count. 
tude, Dedekind considered the cuts through the system which divide it into two parts, lying to the right and left of the cut respectively. Wherever there was a "gap" caused by the cut-i. e., neither a last element before the cut nor a first element after the cut-he filled in the gap with an irrational number. A definite type of order (the "order-type" $\theta$ ) is thus obtained for the continuous number system created. In accordance with this analysis a continuous interval becomes merely an ordered aggregate of separate points, non-countable in number because the gaps in the system of rational points in their natural order were shown by Cantor to be noncountable. Hence the mathematical continuum or matrixand, if you so will, the physical ether to correspond-is resolved into separate but ordered constituents with a reality and discreteness comparable to that of a row of marbles extending infinitely far to right and left.

It is worthy of remark that this "arithmetization" of the continuous interval is in everyway parallel to the recent progress of physics, which tends more and more to become a theory of discrete molecules, atoms, and electrons. Even the existence of the matrix or ether in which these are imbedded has become problematical and open to suspicion. To the atomic structure of a continuous region, if I may call it that, the mathematician also has been driven by the necessity for clear thinking. The question whether anything can be gained for physical theory by supposing our continuous space or ether to be characterized by a higher and more complex order-type has, so far as I know, not been even broached. Within our present continuous space the point-set theory enables us increasingly to consider different groupings of points. In his Houston Lectures Borel has remarked how different physical theories require and engender different mathematical theories. Thus the differential calculus tallies with a physical theory of continuous matter. It seems by no means a wild fancy that because of its essentially atomic structure the point-set theory may develop into an indispensable tool of an atomic physics.

The analysis of the continuum is closely connected with one of the characteristics of modern geometrical development, the search for an axiomatic basis. While too much should certainly not be claimed for the point-set theory in this regard, it is probably not too much to say that it has given, in a 
general rather than a specific way, a strong impulse to this search, though, of course, one entirely secondary to the impulse imparted by the non-euclidean geometry. Yet even more than the latter, it involves a most careful critique of geometrical relations. Historically considered, it is difficult to believe that Hilbert's Foundations of Geometry could have been written before a considerable development of point-set theory. Be this as it may, the rôle of the theory becomes clear and explicit in one of Hilbert's later communications* on the Foundations of Geometry which lays the foundation for geometrical motion.

In this memoir Hilbert achieved the solution of a problem of non-euclidean geometry proposed long before by Riemann, who (with Helmholtz) was the first to attack this subject upon the analytical side. Taking space as the "Zahlenmannigfaltigkeit" of analytical geometry, Riemann asked what properties must be imposed upon this number-spread to give it the character of an ordinary geometry, though not necessarily a euclidean one. His fundamental concept and tool was the element of arc, to which his axioms relate. But to build a finite geometry upon the element of arc, integration is necessary. $\dagger$ Now neither the notion of integration nor of an element of arc is fundamentally simple. Hence his solution, as also for other reasons, cannot be regarded as satisfactory. Later the problem was attacked by Lie, whose attention had been called by Klein to the fact that the notion of a continuous group was involved in both Riemann's and Helmholtz's work on non-euclidean geometry. Lie's axiomatic handling of the problem by the use of the continuous group is also very far from elementary, since in defining the group he employs only transformations which are represented by differentiable functions. Also for the same reason his results are restricted in generality.

Such was the condition of the problem when it was attacked in 1902 by Hilbert. His communication is in his best style and has the elegance always characteristic of his geometrical work. He lays down just three axioms of motion, which I shall not stop to explain but which may be expressed tersely but vaguely as follows:

1. The movements of the plane into itself form a group.

* Math. Ann., vol. 56 (1902), p. 381.

$\dagger$ Cf. Lie-Engel, Theorie der Transformationsgruppen, vol. 3, p. 395. 
2. Every ("wahre") circle contains infinitely many points.

3. The movements form a closed system.

A geometry based on these axioms is then proved to possess the character of either the euclidean geometry or the hyperbolic geometry of Bolyai and Lobachevsky. In the proof Hilbert sloughs off entirely such unnecessary elements as differentiability and even the employment of functions.

The memoir is a splendid interweaving of the basal concepts of two great subjects, the group and point-set theories. From almost the first line to the last it is saturated with the notions and methods of the point-set theory. I know of no more beautiful application of the theory to be found anywhere. I have chosen it therefore as a peg on which to hang some of my remarks.

First of all, I wish you to notice that the point-set theory must enter indispensably into problems like this, which deal with the foundations of geometry and seek the most general conditions under which our space theorems and assumptions are to hold. For when the axiomatization of geometry passes beyond its simpler stages, we must begin to consider infinite aggregates of points, duly grouped and arranged. Hence point-set theory implicitly or explicitly begins to enter. We have not merely to decompose a theorem or assumption into simpler fundamental elements and relationships, but in the synthesis which follows we have also to combine into sets all points or curves which fulfil certain conditions. Thus when Hilbert considers all possible motions of a plane into itself which leave a particular point unaltered, he must show that the different positions taken by any other point form a set of points infinitely dense in themselves and closed, in other words, a perfect set; that this perfect set has the order type of the linear continuum, consisting of an ordered countable set of points dense in itself and their limit points; that this linearly ordered set is a closed Jordan curve containing in its interior the fixed point of the motion; that these Jordan curves of motion enclose one another like the set of concentric circles; and so on. In this manner he demonstrates that in any motion of the plane which leaves a fixed point invariant, the path described by any point possesses all the essential characteristics of a circle viewed in the light of analysis situs.

Furthermore, Hilbert's problem is typical of an ever increasing number of problems in which we have to settle the 
exact geometrical character of a set of points, the locus being often constructed point by point through some analytic process with a final combination of them into a whole. Frequently the condensation method is applied, of which I shall speak later. The problems may differ widely in character, ranging from the consideration of such abstract loci as Hilbert here obtains to the determination of the nature of a dynamical trajectory or of the set of singularities of an analytic function.

A third aspect of Hilbert's work which I wish to emphasize is that it belongs to the province of analysis situs. The plane with which he is dealing is defined as a system of points or "Dingen" which can be referred by a one-one correspondence to the finite number plane or a portion (Teilsystem) of the same. Some of you have doubtless been already asking what is to be understood by the term "movement of a plane" into itself which I have been using. By Hilbert's definition this is any continuous 1-1 transformation of the number image of the plane into itself which leaves unaltered the sense of a circuit around any Jordan curve of this image-plane. Hence by very definition the problem is one of analysis situs. The increasing importance of this branch of mathematics need scarcely be argued here. Ever since Riemann revealed the importance of the "genus" or "class" for a Riemann surface and for the corresponding functions, the qualitative distinctions of analysis situs have proved to be fundamental for the detection of differences in analytic or geometric theories and for their differentiation one from another. Now it is this province of mathematics which is today being invaded by the two allies, the function-theory and the theory of point-sets, and so successfully has it been invaded that its independence has been almost lost.* It remains only to determine which of the two allies will annex it or how they will divide the spoils. In his report at the Zurich International Congress (1897) on the "Development of the general theory of analytic functions in recent time" Hurwitz stated "die Aufgabe der Analysis Situs" to be this, "die Invarianten der einzelnen Klassen von Punktmengen auszusuchen." I wish to preserve my neutrality, but I venture nevertheless to interpret this as an early prediction of the annexation of analysis situs by the theory of point-sets. As examples of invariants of analysis

* However, one large division of the subject has been scarcely affected by the methods or ideas of point-set theory. 
situs which are unaltered by any continuous 1-1 point transformation, I may cite such concepts as limit-point, a perfect point-set, the power of a point-set, and the accessibility or inaccessibility of a specified point of the boundary of a region by means of a path from any interior point which, save for its terminal boundary point, lies wholly in the interior of the region. Examples of invariantive properties are expressed in the familiar theorem that a Jordan curve divides the plane into two regions, also the theorem of Brouwer that the number of dimensions of a space is unaltered by a continuous 1-1 transformation; and so on.

The enrichment of geometry by the theory of point-sets is by no means confined to the department of analysis situs. The new theory has shown itself strong-I might almost say unexpectedly strong - on the quantitative as well as on the qualitative side. As early as 1882 an effort was made by Hankel to attribute content to a discrete set of points. This concept contributed a new element to the growth of the theory. In the more comprehensive form of "measure" devised by Borel and Lebesgue it becomes a powerful agent for quantitative discrimination between sets of points. Thus the rational points in an interval are given a measure 0 and in consequence become negligible in comparison with the irrational points, whose total measure is equal to the length of the interval. With this keen-cutting instrument, measure, Lebesgue has sharpened Riemann's definition of an integral or, more exactly stated, has fashioned a new integral to take its place, far more comprehensive and for theoretical purposes much more powerful. It is true that the new integral has been little applied as yet in geometry, but its usefulness in analysis leaves little doubt that it must eventually become important in geometry also. Such intuitive ideas as the length of a curve or the area of a surface involve much difficulty as soon as we leave the simpler cases, and require the use of measure or other point-set analysis for their development. By point-set means various species of curves have been distinguished, such as Jordan curves, rectifiable and non-rectifiable curves, space-filling curves, the Lebesgue-Osgood curves with areal content, and so on. I may also mention Lebesgue's developable surfaces which are not applicable to a plane, and applicable surfaces which are non-developable. These specifications will suffice to indicate the enrichment of geometry through new ideas. A large num- 
ber of the familiar geometrical concepts, such as the instantaneous curvature of a curve or a surface, were brought in through the differential calculus and begot a differential geometry. Is it too much to expect that new ideas of a totally different import will cause the creation of a corresponding geometry?

Taken as a whole, the point-set theory is conspicuously and characteristically free from formulas. It strikes beneath the formula, which usually limits by its concreteness and definiteness. This absence of equations and formulas is an indication both of strength and of weakness. The sword cuts both ways. The point-set theory becomes less useful as the problem becomes more restricted and regular, in particular when the domain is a continuum and the problem falls under the differential calculus in which the functions are so highly civilized as to be both continuous and differentiable. At first thought there might be some surprise at the almost complete absence of point-set considerations in the differential geometry. I was'much interested to find that one of my well-informed colleagues had thought of point-set theory as a tool especially adapted for use "im Kleinen"- to use a significant term of Osgood. To me, on the other hand, it had appealed because of its power "im Grossen." In its essential nature it seems to me more allied to integration than to differentiation. It is, of course, true that such concepts as limit point or point of condensation in the Lindelöf sense* apply not merely im Kleinen but im infinitesimalen Kleinen; that is to say, when the region or interval containing the point must be indefinitely contracted. The most characteristic point-set methods also involve an analysis im Kleinen. On the other hand, the greatest concepts of the theory, such as countability, derived set, perfect set, are concepts im Grossen. Thus while in the two latter we consider the limit points of the set, our attention is turned to the totality of such points. Similarly, the most common point-set method is one by which a property im Kleinen is first established over a dense sub-set of points and then extended to all points of the set. I doubt not that much of the characteristic strength of the point-set theory lies precisely in this union of consideration $\mathrm{im}$ Kleinen and im Grossen. But the latter is the dominant factor conspicuously evinced in the applications. We are,

* A point in whose vicinity there is an uncountable number of points of the set. 
in fact, no longer content with study im Kleinen and to see in part. Modern mathematics demands, and demands with increasing insistence, methods which give control over the whole of the object under consideration, whether it be function or geometrical figure. The point-set theory complies with this demand. We have passed through a differential period in the development of mathematics. To what extent this will be succeeded by a point-set period it remains for the future to disclose.

I have said that the theory has had little or no application in the differential geometry of curves and surfaces. I must not, however, omit to mention one great application to geodesics upon surfaces of negative curvature made by Hadamard in a memoir* wonderful for the extensive qualitative results obtained with most sparing use of formulas. His attention is not confined in the usual manner to a single geodesic curve but he considers these curves in their totality. As in Poincaré's work on differential equations, so here success is obtained because he dares the whole. The surface of negative curvature may have any number of nappes stretching out to infinity, and may be pierced, somewhat like an anchor ring, with any number of holes. Four kinds of geodesics are distinguished: (1) closed geodesics and (2) those which are asymptotic to these, (3) geodesics which lie in finite space but are neither closed nor asymptotic to closed geodesics, and (4) geodesics which run off to infinity. If the connection of the surface is greater than 2, there is a countably infinite number of closed geodesics, and they form a set "condensed in itself," to apply a term of Hadamard which is not quite self-explanatory. $\dagger$ But the most interesting result relates to the set of geodesics which pass through any point of what is termed the "finite part" of the surface. The directions of those geodesics through the point which do not go to infinity form a perfect set of directions nowhere dense, the interior of the intervening sectors being filled with geodesics which run to infinity. Thus the notion of a perfect set nowhere dense has here its analogue in the set of directions at a point which belong to geodesics of the first three species.ł

\footnotetext{
* Liouville's Journal, ser. 5, vol. 4 (1898), p. 27.

$\dagger$ The exact meaning is explained on p. 55 of his memoir.

¥ Point-set considerations of other character enter into the investigations of geodesics upon closed polyedral surfaces by Stäckel and Rodenberg; Rendiconti del Circolo matematico di Palermo, vol. 22 (1906), p. 141, and vol. 23, pp. 107, 111.
} 
This work of Hadamard is the last specific example which I shall cite of the influence of point-set concepts upon geometrical theory, and in some ways it is the most remarkable one because the notion of a perfect set nowhere dense is not only carried over without modification from a set of points to a set of curves but is actually and unexpectedly realized, not through some artificially constructed example but in practical study. The example emphasizes the importance of the extension of point-set theory to sets of curves. For this a further development is necessary. Fréchet has done pioneer work in laying foundations for a theory of infinite sets of objects other than points, and I may also refer to the investigations of Arzelà, Moore, and others. The incipient geometry of infinitely many variables is also closely related and seems destined to have a very considerable importance. But what seems particularly desirable is concrete application and concrete study of sets of curves because of the broad field of application in geometry and dynamics. In this connection I should not forget to refer to Volterra's work on differentiation with respect to a curve, which involves point-set considerations to a much greater degree than does the ordinary differential calculus. But while reference to it here as an outcome of point-set theory should not be omitted, I regard it, more strictly considered, as forming a distinct branch of mathematics, lying like the differential calculus, just without or within the point-set theory according to one's point of view.

The service of the point-set theory to geometry has not by any means consisted solely in its fertilization of geometry through new ideas, but almost equally in its introduction of new methods. Time altogether fails me to enter adequately into this aspect of the subject, and it is less necessary because the methods have been in some slight degree implied in preceding considerations and are involved still more in those which follow. I should like, however, to note incidentally that the nature of a point-set entering into a problem often prescribes the method to be employed. I shall content myself with a single explicit example of one of the most typical methods, which I have already termed the condensation method. This is taken from Hilbert's* proof of the existence of geodesics upon surfaces of analytical character.

* Jahresbericht der Deutschen Mathematiker-Vereinigung, vol. 8 (1899), p. 185. Cf. also Noble's Dissertation, Göttingen, 1901. 
The length of a geodesic line upon the surface is presented under the form of an integral between the two given but arbitrary points $A$ and $B$ and is to be minimized by proper choice of the surface curve between the two points. The problem is therefore also one of the calculus of variations. The crucial point is to demonstrate, under the conditions given, the actual realization of the lower limit of this integral by some curve on the surface between the two points. To establish this Hilbert selects a sequence of curves between $A$ and $B$ whose lengths approach indefinitely this lower limit. On each of these curves the middle point is taken. A limit point of these middle points gives then a first point $P_{1 / 2}$ of the desired geodesic. The curves between $A$ and $P_{1 / 2}$ and between $P_{1 / 2}$ and $B$ are then considered in exactly the same way, and two new limit points $P_{1 / 4}$ and $P_{3 / 4}$ are obtained which are points of the final geodesic. By continuing in this manner an ordered, dense, and countable set of points $P_{n / 2 m}$ is obtained whose derived set is shown to be a curve between $A$ and $B$, the length of which is equal to the lower limit of the lengths of all possible curves on the surface between the two points. It is therefore the required minimum line or geodesic. By the same general method the fundamental and well-known existence theorem of Hilbert in the calculus of variations has been established.* These are but samples of geometrical problems in which the condensation method would appear to be almost the only resort. The method is easy and strong because it grips the problem beneath the formula close to the roots and seems especially adapted to the calculus of variations, in which the point-set theory has already played a considerable part.

From the calculus of variations and the study of geodesics it is not far to the applications in dynamics. Geodesics are indeed a special case of dynamical trajectories. In these applications the methods of point-set theory have, I think, exerted a greater influence than its concepts, but it is not always possible to separate them. The first noteworthy dynamical application of which I am aware was made by

* Hilbert illustrated " the gist of his method by the example of the shortest line upon a surface," and a further development of the method was given by Noble (loc. cit.) and by Hilbert in his lectures. Important extensions are due to Bolza, Lebesgue, and Carathéodory. For a general resume see Bolza's Lectures on the Calculus of Variations, p. $245 \mathrm{ff}$.; also Carathéodory, Math. Ann., vol. 62 (1906), p. 493. 
Poincaré in his study of dynamical stability.* The motion of a particle or system was said by him to be stable à la Poisson when it returns an infinity of times within any prescribed distance of its initial position, however small. This is the case if the motion is periodic but can also be true when the motion is not closed and the system never returns exactly to the initial position. In celestial mechanics, the motion or transformation of space is often one which preserves volumes, thus resembling the motion of an incompressible fluid in hydrodynamics. This is presupposed by Poincaré. If the motion is continuous, we may obtain a discrete transformation by considering the change which takes place in some fixed but arbitrary time interval $T$. Suppose now the volume to be limited and consider the result of applying the transformation repeatedly to any initial part $U_{0}$ of the volume. Since the transformation by hypothesis preserves volume and the total volume is limited, the iteration of the transformation must bring this part into some position overlapping its original position $U_{0}$. Suppose this to occur after $\alpha_{1}$ iterations, i. e., at the time $\alpha_{1} T$. Let $U_{0}^{(1)}$ be the part common to the two overlapping volumes and apply the transformation in similar manner to this part. By the same argument it must come to overlap itself, say after a time interval $\alpha_{2} T$. Let $U_{0}{ }^{(2)}$ be the common part, and apply the transformation again to this part. Continuing in this manner, we obtain a sequence of volumes $U_{0}^{(n)}(n=0,1,2, \cdots)$, contained each in the preceding. Now by the well-known theorem of pointsets which is the basis of the familiar method of the "Einschachtelung der Intervalle oder Bereiche," there must be at least one point common to a sequence of closed sets contained each in the preceding. If the successive pieces $U_{0}{ }^{(n)}$ do not grow indefinitely small with increasing $n$, we may confine our attention at each successive stage of the reasoning to as small a portion of the overlapping volume as we please and thus obtain a series of volumes contracting to a single limiting point. Take this common point, and beginning de novo, apply to it the inverse transformation, thereby reversing the direction of motion of the point along its path. Since the point lies in $U_{0}^{(n+m)}$, it is easy to see by the reversal of the preceding considerations that it must lie in $U_{0}{ }^{(n)}$ after the lapse of any time interval of the form

* Les Méthodes nouvelles de la Mécanique céleste, vol. 3, chaps. 26 and 27; in particular, sec. 291-6. 


$$
\text { - } T\left[\alpha_{n+m}+e_{m-1} \alpha_{n+m-1}+\cdots+e_{2} \alpha_{n+2}+e_{1} \alpha_{n+1}\right],
$$

where each $e_{1}, e_{2}, \cdots, e_{m-1}$ is either 0 or 1 . As $U_{0}^{(n)}$ may be taken as small as we wish by choosing a sufficiently large $n$, it follows therefore that the point returns an infinity of times to the vicinity of its initial position, and hence its motion is stable à la Poisson.* Hence in any volume there is one, and consequently an infinite number of points whose motion is stable in the inverse transformation; also for like reason an infinite number in the direct transformation (or in both).

The question next arises whether these points of stable motion should be considered as the rule or the exception. Poincaré therefore goes on to prove that the probability of unstable motion for a particle is infinitely small. The term probability, of course, requires definition. It suffices here to say that the probability that a molecule taken at random in volume $V$ will lie at a given instant in a given portion $U$ of that volume is $U / V$. In terms of Lebesgue's measure Poincaré's conclusion can be reformulated as follows: The set of points whose trajectories are not stable has a measure 0, and accordingly the probability that a point will not return infinitely often to the vicinity of the initial position is 0 .

This conclusion can be extended from particles to systems with $n$ degrees of freedom, provided that in the corresponding $n$-dimensional parameter-space there is a volume or other invariant integral with positive integrand. Upon this result as a foundation rest other theorems of Poincaré which lie without my subject. Thus, though point-set considerations have entered but little into his work on celestial mechanics, they serve to establish a most pivotal result. Their introduction was, I believe, inevitable and necessitated by the very nature of the conclusion. But before discussing this, I wish to place beside Poincaré's result another illustration taken also from celestial mechanics.

One of the fundamental problems of secular variations is that of the existence of a so-called "mean motion" for the perihelion or node. Much has been writen upon the subject since the time of Lagrange, but as it now turns out, prac-

* Poincaré fails to contract the regions $U_{0}(n)$ and therefore does not strictly prove the stability. "Plus exactement," he states, "il y aura des molécules qui traverseront une infinité de fois ce volume." 
tically all subsequent deductions have been erroneous. Mathematically, the problem is as follows: Given two real series

$$
\begin{aligned}
& r_{1} \sin \left(g_{1} t+h_{1}\right)+r_{2} \sin \left(g_{2} t+h_{2}\right)+\cdots+r_{n} \sin \left(g_{n} t+h_{n}\right), \\
& r_{1} \cos \left(g_{1} t+h_{1}\right)+r_{2} \cos \left(g_{2} t+h_{2}\right)+\cdots+r_{n} \cos \left(g_{n} t+h_{n}\right),
\end{aligned}
$$

or the equivalent complex series,

$$
\sum_{m=1}^{n} r_{m} e^{i\left(g_{m} t+h_{m}\right)} \quad\left(r_{n}>0\right) ;
$$

to determine when it is possible to express their sums in the form $r \sin \omega, r \cos \omega$, and $r e^{i \omega}$ respectively, in which the angle $\omega$ is expressible under the form $c t+X(t)$ where $c$ is a constant and $X(t)$ is a limited function of $t$. Lagrange gave two sufficient conditions for this,* and remarked that "outside of these two cases it was very difficult and perhaps impossible to pronounce in general on the nature of the angle." Recently the problem has been completely treated for $n=3$ by Bohl, $\dagger$ who shows that when the condition of Lagrange fails to hold, the determination depends upon two quantities,

$$
\rho=\frac{g_{2}-g_{1}}{g_{3}-g_{1}} \text { and } \zeta=\frac{1}{\pi}\left(\omega_{3}+\rho \omega_{2}\right),
$$

in which $\omega_{1}, \omega_{2}, \omega_{3}$ denote the angles of a triangle with sides $r_{1}, r_{2}, r_{3}$. Now from Lagrange's work it was known that the mean motion exists when $\rho$ is rational. Bohl shows further that for every pair of values $(\rho, \zeta)$, the ratio $\omega / t$ has for $t=+\infty$ a limit $c$, but that, on the other hand, there is a set of values everywhere dense in the region $|\rho|<1,|\zeta|<1$, for which the remainder $X(t)$ is not limited and for which accordingly there is no mean motion. Since also the points $(\rho, \zeta)$ with commensurable $\rho$ are everywhere dense, Bohl concludes that it will be impossible ever to ascertain by experimental data whether or not the mean motion exists. So far as I have noticed, Bohl does not actually use point-set theory in his proof, though sets of number-points frequently appear. More recently F. Bernstein $\ddagger$ has shown by point-set methods

* Namely, that some one of the $r_{i}$ should be greater than the sum of all the others, or $n=2$.

$\dagger$ Crelle's Journal, vol. 135 (1909), p. 189.

$\ddagger$ Math. Ann., vol. 71 (1911-12), p. 417 . 
that the total set of points $(\rho, \zeta)$ for which the mean motion exists has a Lebesgue measure 0 . Bernstein then advances a philosophical or physical principle in which he asserts that in physical measurement we can never take account of a set of points of measure 0 ; consequently, for us there can be no mean motion except in the Lagrangian cases.

Into a discussion of this interesting but questionable principle I shall not enter. What I do wish to do, however, is to point out that both this result and that of Poincare are without doubt typical of the exceeding complications of physical nature which must increasingly engage the mathematician's attention. Thus, for example, orbits possessing a specified character and those which do not, may be tangled densely together in the space of the parameters on which these orbits depend. It is to the point-set theory that one must turn to control the two sets of parameters both quantitatively and qualitatively. Already groups of exceptional points of measure 0 have been discovered with considerable frequence in the theory of functions. By allowing now for such a set of exceptional points in an interval or field we can often obtain the necessary or sufficient condition for a desired result, for instance in the case of Fourier's series. Prior to the introduction of the Lebesgue measure no general theorems were visible because when the cases pro and the cases con were both infinite in number, there was no means of overcoming the difficulty and reaching a definite result. Undoubtedly, also, we must expect to control many phenomena in astronomy and dynamics only by admitting a set of exceptions of measure 0 .

The introduction of measure also broadens the scope of the theory of probability, which is so fundamental to a large part of physical speculation. For by its use account can be taken of infinite sets of discrete points, dense and complicated; and more generally, exceptional cases, infinite in number, can be brought under the theory of probability by attributing to them a probability proportional to the measure of the corresponding points in the space of the parameters upon which they depend, though care must be taken inasmuch as measure is not an invariant of analysis situs and hence may be dependent on the parameters introduced. This application of measure is as yet prospective rather than actual. In this connection I should mention also Borel's treatment of probability for a countably infinite number of cases. 
Two other aspects of the above discussed work of Poincaré seem to me to be also worthy of attention. In the first place, to get a grip upon dynamical stability Poincaré abandons the infinitesimal transformation and considers instead the result of iterating a finite transformation arising in time $T$. From a single point or an arc traversed in time $T$ there arises by iteration of the transformation a whole set of points or arcs, which are capable of point-set treatment. A wide field is opened in this manner for the future application of point-set methods, especially in dynamics and the theory of geometric transformations. As an instance of application to the latter subject I may cite my determination* of the form of a oneparameter group of projective transformations when a single finite substitution is given instead of the generating infinitesimal substitution of Lie. I need not plead the prospective usefulness and importance of this iterative point-set method with those of you who heard Professor Birkhoff's paper at our last summer meeting. But I should also add that in his memoir on the movement of dynamical systems $\dagger$ he has obtained some notable results on dynamical stability by what is essentially direct point-set treatment of the infinitesimal transformation.

The remaining aspect of Poincarés transformation which I wish to recall is its assumed property of conserving volume. Later, in his last published memoir, $\ddagger$ Poincare put before the mathematical world an unproved theorem based on a similar restriction. If, namely, a circular ring is changed into itself by a continuous 1-1 transformation with preservation of areas and in such a way that the points of the one boundary are moved clockwise and those of the other boundary anti-clockwise, there must be at least two invariant points within the ring. Clearly, this is a problem of analysis situs, but with an accessory condition of preservation of areas which makes it at the same time a problem of hydrodynamics. Professor Birkhoff's $\S$ brilliant solution is, I hope, known to you all. His method contains a highly ingenious but, as he himself would recognize, somewhat artificial device. The natural attack seems to me to be through point-set methods, as was

* Transactions, vol. 13 (1912), p. 353.

$\dagger$ Bulletin de la Société Mathématique de France, vol. 40 (1912), p. 305.

\$ Rendiconti del Circolo matematico di Palermo, vol. 33 (1912), p. 375.

$\S$ Transactions, vol. 14 (1913), p. 14. 
apparently the thought of Poincaré. Results of similar import in analysis situs, but without the restriction of the preservation of volume, have been attained through point-set considerations by Brouwer, who shows, for example, that every continuous 1-1 transformation of a sphere of even dimensions into itself without reversal of circuit sense, or of a sphere of odd dimensions with such reversal, leaves at least one point invariant. It may be confidently expected that many results in hydrodynamics will be obtained when the requirement of invariability of area or volume is added.

I turn now from celestial mechanics and hydrodynamics to the field of statistical mechanics. Consider a so-called gas-model, composed of a very large number $N$ of molecules having each $r$ degrees of freedom. The corresponding canonical equations of Hamilton,

$$
\frac{d q_{s}{ }^{(k)}}{d t}=\frac{\partial E}{\partial p_{s}{ }^{(k)}}, \quad \frac{d p_{s}{ }^{(k)}}{d t}=-\frac{\partial E}{\partial q_{s}{ }^{(k)}} \quad \begin{aligned}
& (s=1, \cdots, r), \\
& (k=1, \cdots, N),
\end{aligned}
$$

involve $r$ generalized coordinates $q_{s}{ }^{(k)}$ and $r$ moments $p_{s}{ }^{(k)}$ and the total energy $E$. The $2 r N$ quantities $q_{s}{ }^{(k)}, p_{s}{ }^{(k)}$ determine a "phase" of the system. For geometrical representation a $2 r N$-dimensional space is required, called the phase space (Phasenraum). Now it was a great discovery of Liouville, made independently a second time by Boltzmann and Poincaré, that in consequence of Hamilton's equations volume must be an invariant integral of motion in this $2 r N$-dimensional space. The given value of the energy $E$ determines what is called the energy-surface within this space, upon which the molecules move in a one-dimensional path. As the foundation of their gas and statistical investigations Boltzmann and Maxwell assumed that the system was "ergodic." By an ergodic system is to be understood one in which a molecule so moves as to pass through every single point of the energy surface upon which it lies. Since, furthermore, there is a unique motion at any point of the phasespace, all the particles upon the energy-surface are describing one after another exactly the same curve. Now the possibility of such motion was assumed but not proved by Boltzmann. Mathematically considered, this is tantamount to the assumption of the existence of a space-filling curve without double points. For if there were anywhere a double point in the path of a particle in the phase-space, the corresponding 
gas-model would pass twice through the same state, and the particle would in consequence begin to repeat its motion without describing the rest of the curve. What would have seemed more improbable than that the freak space-filling curves first invented by Peano could ever have a practical bearing! Imagine the scorn of the average physicist for such impractical, crazy, mathematical pathology. Yet here is a master physicist Boltzmann not merely postulating the existence of space-filling curves upon his energy-surface but making an impossible assumption, and therefore an actually crazy one, that such curves can exist* without double points. The proof of their non-existence belongs to the point-set theory and results from Brouwer's theorem $\dagger$ that it is impossible to build two regions of different dimensions-in the present case $\ddagger$ the $(2 r N-1)$-dimensional space-surface and the one-dimensional time-axis-one upon the other by means of a continuous 1-1 transformation.

The impossibility of an ergodic system has been considered also from another standpoint by Plancherel, $\S$ who shows that the path of a particle has a surface measure 0 and hence cannot fill the energy-surface as desired. It follows immediately that the number of different path-curves on the surface must be more than countable. $\|$ I may mention further that Rosenthal II has replaced Boltzmann's supposition by a quasiergodic hypothesis, according to which each path on the energy-surface comes within any prescribed distance of every point of the surface.

In essence the statistical mechanics is a universal application of averages or, in other terms, of a theory of probability. It is therefore to be anticipated that numerous other applications will be here made of the theory of point-sets.

The examples which I have brought to your attention are, I trust, sufficient to convince you that the point-set theory has vital relations to the study of dynamical trajectories as well as to geometry. I have preferred to illustrate its rôle with concrete examples rather than to indulge in abstract

* Cf. Rosenthal, Annalen der Physik, vol. 42 (1913), p. 796.

$\dagger$ Math. Ann., vol. 70 (1911), p. 161 .

† For this case the simple proof given by Jürgens suffices; cf. Jahresbericht der Deutschen Mathematiker-Vereinigung, vol. 7 (1898), p. 54.

$\S$ Annalen der Physik, vol. 42, p. 1061.

If This is also shown by Rosenthal (1. c.) who employs for that purpose

Baire's concept of point-sets of the first and second categories.

II Annalen der Physik, vol. 43 (1914), p. 894. 
discussion. In so doing we have dug down to the very roots of geometry and dynamics but we have also touched the branches where the shoots are pushing out. Too much must not be claimed. Yet if the point-set theory is not the sap of the tree, it is at least one of its most indispensable ingredients. The usefulness of any mathematical theory must be determined not by its isolation but by its ability to combine with other theories. In this the point-set theory has shown itself most elastic. Had I more time I would attempt to show you that it offers many of the same advantages as analytical geometry. While strong for analysis and decomposition, it is equally strong on the constructive side. Complex groupings of points are made simple, and the way is thus prepared for new discovery. And above all, in its development the arithmetization of analysis is kept close to geometrical intuition.

In tracing the service of the theory of point-sets in geometry and dynamics, we have found only in part achievement, in part present evolution and promise. But it is precisely because of this mocking incompleteness that I have chosen for my topic today the rôle of the point-set theory in geometry and dynamics, trusting that for you also this will be its lure.

UNIVERSITY OF WISCONSIN, MADISON, Wis.

\section{AN ENUMERATION OF INTEGRAL ALGEBRAIC POLYNOMIALS.}

BY PROFESSOR A. B. FRIZELL.

(Read before the American Mathematical Society, January 1, 1915.)

THE proof given by Weber* that the algebraic numbers form a countable set orders them according to the values of a certain function of the coefficients in their defining equations. The present note suggests a more direct enumeration of these equations. The algebraic polynomials $\sum_{i=0}^{n} a_{i} x^{n-i}$ in which all coefficients are natural numbers can be put into one-to-one correspondence with the set of natural numbers by the fol-

\footnotetext{
* Algebra, Bd. II, p. 824.
} 